\title{
BMJ Open Fertility outcome analysis after surgical management of tubal ectopic pregnancy: a retrospective cohort study
}

\author{
Jingwei Li, ${ }^{1}$ Kailei Jiang, ${ }^{2}$ Fujie Zhao ${ }^{1}$
}

To cite: Li J, Jiang K, Zhao F. Fertility outcome analysis after surgical management of tubal ectopic pregnancy: a retrospective cohort study. BMJ Open 2015;5:e007339. doi:10.1136/bmjopen-2014007339

- Prepublication history for this paper is available online. To view these files please visit the journal online (http://dx.doi.org/10.1136/ bmjopen-2014-007339).

Received 2 December 2014 Revised 13 March 2015 Accepted 18 March 2015

\section{ABSTRACT}

Objectives: To compare the subsequent fertility and risk of recurrence of an ectopic pregnancy (EP) in women who had had an EP, according to the type of surgical treatment they received - that is,

salpingectomy, salpingostomy or tubal anastomosis. Methods: A retrospective cohort study was carried out between January 2003 and September 2011 of 618 patients admitted to hospital with tubal EP and who had received surgical treatment (salpingectomy, $\mathrm{n}=434$; salpingostomy, $\mathrm{n}=112$; and tube anastomosis, $\mathrm{n}=72$ ). Main outcomes included the first intrauterine pregnancy (IUP) and recurrent EP.

Results: The crude IUP rates up to 24 months after surgery were $55.5 \%$ for salpingectomy, $50.9 \%$ for salpingostomy and $40.3 \%$ for tubal anastomosis treatments. In the multivariate-adjusted model, with the patients receiving salpingectomy as the reference group, HR for patients after salpingostomy and tubal anastomosis treatments for IUP were $0.912(95 \% \mathrm{Cl}$ 0.762 to 2.017$)$ and $0.619(95 \% \mathrm{Cl} 0.328$ to 0.927$)$, respectively. The 2-year cumulative recurrent EP rates were found to be $8.1 \%$ for salpingectomy, $6.3 \%$ for salpingostomy and $16.7 \%$ for tubal anastomosis treatments. Taking the patients receiving salpingectomy as the reference group, the patients who received tubal anastomosis had a positively higher risk of recurrent EP ( $\mathrm{HR}=2.280 ; 95 \% \mathrm{Cl} 1.121$ to 4.636$)$ in univariate analysis. Adjustment for other potential confounders only slightly attenuated the HR.

Conclusions: The patients with an EP receiving tubal anastomosis treatments appeared to have a lower 2-year rate of IUP and a higher risk of recurrent EP after adjustment for other potential risk factors.

\section{INTRODUCTION}

The incidence of ectopic pregnancy (EP) has increased to a level of $2 \%$ in developed countries and may seriously affect women's health and future fertility. ${ }^{1}{ }^{2}$ During recent decades, the diagnosis and efficacy of treatment of EP has progressed significantly. ${ }^{3-5}$ Surgical intervention has long been the 'gold standard' for treatment of EP, although the medical management of unruptured EP is a safe and effective alternative. ${ }^{3}$ Whichever

\section{Strengths and limitations of this study}

- Controversy still exists about the role of surgical treatment in optimising subsequent fertility for patients with ectopic pregnancy (EP). This study evaluated the difference between tubal anastomosis and other types of surgical treatment for $\mathrm{EP}$ in China.

- Patients with EP were recruited from one hospital, which might have led to selection bias and limited external validity of the findings.

- This was a retrospective observational study and a randomised clinical trial is required to validate our findings.

treatment is used, in addition to its effectiveness, must preserve patients' fertility, and limit the risk of recurrence.

Surgical treatment for EP includes salpingectomy, salpingostomy and tubal anastomosis. However, controversy still exists about the role of surgical treatment in optimising subsequent fertility, which has been examined by previous studies. ${ }^{6}{ }^{7}$ Fertility after salpingectomy has been compared with salpingostomy in many retrospective studies or reviews. ${ }^{7}$ In a population-based study, the crude cumulative rates of intrauterine pregnancy (IUP) differed according to the treatment method in univariable analysis $(p=0.0079)$, with a lower fertility after radical treatment compared with conservative treatments. However, the results were not statistically significant after adjustment for confounders. ${ }^{8}$ In addition, the risk of recurrence according to the surgical techniques is still uncertain, with some retrospective studies showing higher recurrence rates after laparoscopic salpingostomy, ${ }^{9}$ and others showing no difference. ${ }^{8} 1011$

Tubal anastomosis restores patency of the fallopian tubes after sterilisation, in patients who wish to become pregnant. Pregnancy rates are between $54 \%$ and $88 \%$ for laparotomy and $31-85 \%$ for laparoscopy. ${ }^{12}$ 
Tubal anastomosis is also used as a method of surgical treatment for EP. However, there are few data evaluating the difference between tubal anastomosis and other types of surgical treatment.

This study aimed to compare the subsequent fertility and risk of recurrence of an EP in women who had had an EP, according to the type of surgical treatment they received-that is, salpingectomy, salpingostomy and tubal anastomosis.

\section{PATIENTS AND METHODS}

\section{Patients recruited}

We retrospectively reviewed patients with a tubal EP who were admitted to hospital for surgical treatment in the department of obstetrics and gynecology in Shengjing Hospital between January 2003 and September 2011. Three choices of surgical approach were available for EP: salpingectomy (removal of the fallopian tube), salpingostomy (incising the tube to remove the tubal gestation but leaving the remainder of the tube intact) and tubal anastomosis (removing abnormal tissue and reapproximating the healthy tubal segments with as little adhesion formation as possible). Indications for surgery included haemodynamic instability; suspicion of, or risk factors for, rupture; contraindications to methotrexate or failed medical treatment. The decision to choose salpingectomy, salpingostomy or tubal anastomosis was based on the pretherapeutic score proposed by Pouly et al. ${ }^{13}$

Before surgery, women underwent evaluation for EP and routine preoperative evaluation and preparation. During surgery, methylene blue was given to check whether or not the contralateral tube was open at operation. All interventions were carried out in accordance with the procedural standards of the participating hospital. All patients seen for EP are admitted to hospital for close follow-up. Patients excluded from this study were those with a prior history of EP, patients who did not report seeking pregnancy during follow-up and were using a contraceptive method, patients who had not wanted a baby or had not attempted to conceive, those who had received bilateral salpingectomy or hysterectomy, patients who had received medical treatment with methotrexate and patients receiving in vitro fertilisation; 767 patients met our inclusion criteria. Of these, 149 patients were not included in the study because they were lost to follow-up $(n=114 ; 14.9 \%)$ or because they were aged $\geq 45$ years. Finally, reproductive outcome was analysed for 618 patients (salpingectomy, $n=434$; salpingostomy, $\mathrm{n}=112$; and tubal anastomosis, $\mathrm{n}=72$ ). The study was approved by the ethical committee of Shengjing Hospital of China Medical University and all patients gave their written consent to participate in the study.

\section{Data collection}

A standard case report form at baseline was developed to assess related information for each woman and included: sociodemographic characteristics; sexual, gynaecological, reproductive and surgical histories; smoking habits; condition of conception (eg, contraception, ovulation induction); characteristics of the EP and treatment procedures used.

To identify persistent trophoblast, serum human chorionic gonadotrophin (human chorionic gonadotropin (hCG), expressed in IU/L) was measured postoperatively until undetectable concentrations were reached in all study groups. Persistent trophoblast is defined as postoperative rising or plateauing of serum hCG concentrations. ${ }^{14}$ Patients were contacted and asked whether they had a new pregnancy up to 24 months, were pregnant again, the outcome of subsequent pregnancies and the use of contraceptives and medical measures related to infertility. All IUPs were taken into account regardless of the outcome. For the fertility study, any recurrence was ignored and the follow-up continued and conversely for the recurrence study. Only the first IUP or the first recurrence was used for this analysis.

\section{Statistical analysis}

Continuous variables are presented as the means and $\mathrm{SD}$ and categorical variables are expressed as percentages. We compared patients' baseline characteristics according to surgical treatment by $\chi^{2}$ tests for categorical variables and one-way analysis of variance for continuous variables. The persistent trophoblast and future fertility outcomes (the 2-year cumulative rates of recurrent EP and spontaneous IUP) were calculated for each of the three surgical treatments. Cox proportional hazards models were used to calculate HRs with 95\% CIs for the associations between surgical treatments and fertility outcomes, with the patients receiving salpingectomy as reference group. Univariate and multivariate analysis were used to evaluate the association between the types of surgical treatment and first IUP or the first recurrence.

In the multivariate model, we adjusted for age, surgical treatment, marital status, smoking habits, previous spontaneous abortion and induced abortion, history of live birth, tubal disease and normal contralateral tube. Next, we repeated the multivariate analysis for a subgroup of patients after exclusion of those with an abnormal tube in the salpingectomy group. The proportionality assumption was evaluated by scaled Schoenfeld residuals and the global fit of the models was evaluated by graphically examining the cumulative hazards function relative to the Cox-Snell residuals. All analyses were performed with SPSS statistical software V.13.0 (SPSS Inc, Chicago, Illinois, USA). A $p$ value $<0.05$ was accepted as indicating statistical significance.

\section{RESULTS}

Treatments were salpingectomy for 434 women, salpingostomy for 112 and tubal anastomosis for 72 . The mean $\pm \mathrm{SD}$ ages of patients receiving salpingectomy, salpingostomy and tubal anastomosis were 29.4 \pm 5.1 years, $28.8 \pm 4.9$ years and $28.1 \pm 6.0$ years $(p=0.116)$, 
respectively. Characteristics of the patients in this study are shown in table 1 . The 114 patients who were lost to follow-up had a baseline age (median: 30.2 vs 29.1 years; $p>0.05)$ similar to that of the patients included in the study $(n=618)$. There was no significant difference in the proportion of patients receiving salpingectomy, salpingostomy or tubal anastomosis between the patients who completed the study and those who were lost to follow-up (434:112:72 vs 76:23:15, p>0.05).

The crude IUP rates up to 24 months after operation were $55.5 \% \quad(\mathrm{n}=241)$ for salpingectomy, $50.9 \% \quad(\mathrm{n}=57)$ for salpingostomy and $40.3 \% \quad(\mathrm{n}=29)$ for tubal anastomosis treatments. The cumulative IUP rates differed according to the surgical treatment methods in univariate analysis $(p=0.049)$, with a lower fertility after tubal anastomosis than after salpingectomy treatment (HR=0.469, 95\% CI 0.285 to 0.772$)$. No statistically significant difference was found in the IUP rates between the salpingectomy and salpingostomy groups $(\mathrm{p}=0.276)$. Adjustment for other potential confounders only slightly attenuated the HR (table 2). In the multivariate-adjusted model, HR for patients with salpingostomy and tubal anastomosis treatments for IUP were $0.912 \quad(95 \%$ CI 0.762 to 2.017 ) and 0.619 (95\% CI 0.328 to 0.927 ), compared with the patients receiving salpingectomy as the reference group (table 2).

Table 3 shows the risk of persistent trophoblast and recurrent EP after different surgical treatments. Persistent trophoblast occurred significantly more often in the salpingostomy $(9.8 \%)$ and tubal anastomosis $(8.3 \%)$ group than in the salpingectomy group (1.8\%) (table 3). The 2-year cumulative recurrent EP rates were found to be $8.1 \%$ for salpingectomy, $6.3 \%$ for salpingostomy and $16.7 \%$ for tubal anastomosis treatments. With the patients receiving salpingectomy as the reference group, the patients who received tubal anastomosis had a higher risk of recurrent EP (HR=2.280, 95\% CI 1.121 to 4.636) in univariate analysis. Adjustment for other potential confounders only slightly attenuated the HR (table 3). There was no significant difference for the risk of recurrent EP between salpingectomy and salpingostomy treatments.
In the next subgroup analysis, 327 patients in salpingectomy group with a normal tube had an increased crude IUP rate $(\mathrm{n}=190,58.1 \%)$ and a similar recurrent EP rate $(n=26,8.0 \%)$. With the patients receiving salpingectomy with a normal tube as the reference group, the multivariate-adjusted HR for patients with tubal anastomosis for IUP and recurrent EP were 0.584 (95\% CI 0.308 to 0.891 ) and 1.653 (95\% CI 1.079 to 4.637 ), respectively. There was no significant difference for the IUP and risk of recurrent EP between salpingectomy and salpingostomy treatments and the multivariate adjusted HRs were 0.947 (95\% CI 0.771 to 2.109 ) and 0.984 (95\% CI 0.629 to 1.985 ), respectively.

\section{DISCUSSION}

EP is one of the important reasons for maternal morbidity and mortality in the first trimester, accounting for a $13 \%$ mortality rate. ${ }^{15}$ The effect of different surgical management strategies on subsequent fertility after tubal EP is still controversial. ${ }^{16}{ }^{17}$ Major findings of our study were that patients with an EP receiving tubal anastomosis treatment seemed to have a lower 2-year rate of IUP and a higher risk of recurrent EP after adjustment for other potential risk factors.

Many retrospective studies compared subsequent fertility after salpingectomy versus salpingostomy. According to some studies, there was no statistical difference in subsequent fertility. ${ }^{18}{ }^{19}$ However, with the development of the laparoscopic technique, a trend in favour of conservative surgical treatment was noted by some retrospective studies. ${ }^{9}$ Turan showed that there is no significant difference in IUP rates up to 24 months between salpingectomy $(65.2 \%)$ and salpingostomy $(60.1 \%)$ groups in younger Turkish women. ${ }^{11}$ One retrospective cohort study found that the 7-year cumulative IUP rate was lower in women who had undergone salpingectomy than in those who had undergone salpingostomy. ${ }^{10}$ In our study, patients receiving salpingostomy seem to have a lower rate of IUP $(50.9 \%)$ than patients receiving salpingectomy (55.5\%). However, consistent with other studies, ${ }^{20}{ }^{21}$ there was no statistically significant difference.

\begin{tabular}{lcccr}
\multicolumn{5}{l}{ Table 1 Clinical characteristics of women with an ectopic pregnancy according to surgical treatment } \\
\hline Characteristics & Salpingectomy & Salpingostomy & Tubal anastomosis & p Value \\
\hline Number & 434 & 112 & 72 & \\
Age (years), mean \pm SD & $29.4 \pm 5.1$ & $28.8 \pm 4.9$ & $28.1 \pm 6.0$ & 0.116 \\
Unmarried, $\mathrm{n}(\%)$ & $98(22.6)$ & $20(17.9)$ & $22(30.6)$ & 0.133 \\
Smoking habits, $\mathrm{n}(\%)$ & $39(9.0)$ & $9(8.0)$ & $7(9.7)$ & 0.920 \\
Previous spontaneous abortion, $\mathrm{n}(\%)$ & $26(6.0)$ & $4(4.2)^{\star}$ & $5(11.1) \#$ & 0.271 \\
Previous induced abortion, $\mathrm{n}(\%)$ & $184(42.4)$ & $30(26.8)$ & $25(34.7)$ & 0.008 \\
History of live birth, $\mathrm{n}(\%)$ & $164(37.8)$ & $28(29.2)^{\star}$ & $22(48.9) \#$ & 0.030 \\
Tubal disease, $\mathrm{n}(\%)$ & $49(11.3)$ & $14(12.5)$ & $11(15.3)$ & 0.616 \\
Normal contralateral tube, $\mathrm{n}(\%)$ & $327(75.3)$ & $72(64.3)$ & $32(44.4)$ & $<0.001$ \\
\hline
\end{tabular}

*Data based on 95 women.

\#Data based on 45 women. 
Table 2 Univariate and multivariate analysis factors influencing intrauterine pregnancy

\begin{tabular}{llllll}
\hline & Intrauterine pregnancy, & HR and 95\%Cl & & \\
\cline { 2 - 5 } Treatment & $\mathbf{n}(\%)$ & Univariate & p Value & Multivariate $^{*}$ & p Value \\
\hline Salpingectomy $(\mathrm{n}=434)$ & $241(55.5)$ & 1.000 (reference) & & & \\
Salpingostomy $(\mathrm{n}=112)$ & $57(50.9)$ & $0.831(0.602$ to 1.156$)$ & 0.276 & $0.912(0.762$ to 2.017$)$ & 0.308 \\
Tubal anastomosis $(\mathrm{n}=72)$ & $29(40.3)$ & $0.469(0.285$ to 0.772$)$ & 0.003 & $0.619(0.328$ to 0.927$)$ & 0.039 \\
\hline
\end{tabular}

Laparotomy microsurgical tubal reanastomosis after tubal sterilisation has been performed for more than 30 years, with recently reported pregnancy rates of $60-91 \% .^{22}$ Koh and Janik ${ }^{23}$ reported a $71 \%$ pregnancy rate during a follow-up of 12 months after laparoscopic microsurgical tubal anastomosis. Tan and $\operatorname{Loh}^{24}$ also reported that the pregnancy rates with this same surgery were $47.4 \%$ ( $<6$ months), $57.9 \%$ (6-12 months), $68.4 \%$ (12-48 months) and $73.7 \%$ ( $>48$ months). The IUP rate of our study was $40.3 \%$, which is lower than the results of other studies. Different population characteristics may partly explain the disparity. In addition, there are few studies that compare the fertility outcomes among tubal anastomosis and salpingectomy or salpingostomy in a similar study. In our study, with the patients with an EP undergoing salpingectomy as reference, those receiving tubal anastomosis had a lower risk of IUP.

In addition, recurrent EP was associated with mortality and morbidity, but the risk of recurrence according to the surgical technique used is still under discussion, with conclusions divided. Some found a higher risk of recurrence after laparoscopic salpingostomy. ${ }^{9}$ For others, the rate of recurrence was similar after salpingectomy and salpingostomy. ${ }^{10} 25$ In our study, the highest recurrent
EP rates were found in the tubal anastomosis group $(16.7 \%)$ and the patients receiving this treatment had a higher rate of recurrent EP after adjustment for other potential risk factors. In addition, EP recurrence rates for surgical treatment with salpingectomy $(8.1 \%)$ and salpingostomy $(6.3 \%)$ were similar.

There are two possible explanations for the lower IUP rate and higher EP recurrence after tubal anastomosis. First, a tubal surgery scar after tubal anastomosis can easily cause stenosis and tubal blockage, which interferes with sperm fertilisation of eggs in the fallopian tube, resulting in blastocyst formation in the fallopian tube. Thus, there would be a lower IUP rate and higher recurrence of EP after tubal anastomosis. Second, other potential risk factors may produce these results. In summary, the mechanisms of lower IUP rate and higher EP recurrence after tubal anastomosis are still unclear and further study is needed.

Some limitations of the study should be considered. First, the patients with an EP were recruited from one hospital, which might have led to selection bias and limited external validity of the findings. The results were further examined in a population-based study. Second, this is a retrospective observational study and additional randomised clinical trials are needed to validate our

Table 3 Univariate and multivariate analysis for persistent trophoblast and recurrent ectopic pregnancy (EP) according to surgical treatment

\begin{tabular}{|c|c|c|c|c|c|}
\hline \multirow[b]{2}{*}{ Treatment } & \multirow[b]{2}{*}{ n (\%) } & \multicolumn{4}{|l|}{$\mathrm{HR}$ and $95 \% \mathrm{Cl}$} \\
\hline & & Univariate & p Value & Multivariate $^{\star}$ & p Value \\
\hline \multicolumn{6}{|l|}{ Salpingectomy ( $n=434$ ) } \\
\hline Persistent trophoblast & $8(1.8)$ & 1.000 (reference) & & 1.000 (reference) & \\
\hline Recurrent EP & $35(8.1)$ & 1.000 (reference) & & 1.000 (reference) & \\
\hline Ipsilateral tube & $16(3.7)$ & 1.000 (reference) & & 1.000 (reference) & \\
\hline Contralateral tube & $19(4.4)$ & 1.000 (reference) & & 1.000 (reference) & \\
\hline \multicolumn{6}{|l|}{ Salpingostomy ( $n=112$ ) } \\
\hline Persistent trophoblast & $11(9.8)$ & 4.613 (2.942 to 7.285$)$ & $<0.001$ & 4.078 (2.113 to 6.959$)$ & $<0.001$ \\
\hline Recurrent EP & $7(6.3)$ & 0.760 (0.328 to 1.760$)$ & 0.522 & 0.983 (0.629 to 1.982$)$ & 0.767 \\
\hline Ipsilateral tube & $5(4.5)$ & $1.179(0.653$ to 1.621$)$ & 0.358 & 1.054 (0.359 to 1.747$)$ & 0.524 \\
\hline Contralateral tube & $2(1.8)$ & $0.697(0.231$ to 1.125$)$ & 0.078 & 0.703 (0.307 to 1.351$)$ & 0.219 \\
\hline \multicolumn{6}{|l|}{ Tubal anastomosis $(n=72)$} \\
\hline Persistent trophoblast & $6(8.3)$ & 3.094 (1.782 to 4.801$)$ & $<0.001$ & 2.981 (1.653 to 4.478 ) & $<0.001$ \\
\hline Recurrent EP & $12(16.7)$ & 2.280 (1.121 to 4.636$)$ & 0.023 & 1.654 (1.080 to 3.718$)$ & 0.047 \\
\hline Ipsilateral tube & 9 (12.5) & 3.782 (1.829 to 4.612 ) & 0.017 & 3.214 (1.507 to 4.731 ) & 0.038 \\
\hline Contralateral tube & $3(4.2)$ & 0.961 (0.457 to 1.501$)$ & 0.814 & 0.980 (0.421 to 1.593$)$ & 0.769 \\
\hline
\end{tabular}

*Adjusted by age, marital status, smoking habits, previous spontaneous abortion and induced abortion, history of live birth, tubal disease and normal contralateral tube. 
findings. Third, we measured the outcomes of IUP and recurrence of EP up to 24 months, but a longer follow-up is needed.

In conclusion, the results of this study suggest that the tubal anastomosis group had a lower rate of IUP and a higher rate of recurrent EP. The choice of surgical treatment should be comprehensively evaluated in clinical practice.

Contributors $\mathrm{FZ}$ designed the study and analysed the data. $\mathrm{JL}$ and $\mathrm{KJ}$ reviewed the literature, organised the writing and wrote the initial drafts. FZ and $\mathrm{JL}$ finalised the manuscript. $\mathrm{FZ}$, $\mathrm{JL}$ and $\mathrm{KJ}$ contributed to the analysis and interpretation of the data, revised the paper critically for important intellectual content and approved the final version to be published.

Funding This research received no specific grant from any funding agency in the public, commercial or not-for-profit sectors.

Competing interests None.

Patient consent Obtained.

Ethics approval The study has been approved by the ethical committee of Shengjing Hospital of China Medical University.

Provenance and peer review Not commissioned; externally peer reviewed.

Data sharing statement No additional data are available.

Open Access This is an Open Access article distributed in accordance with the Creative Commons Attribution Non Commercial (CC BY-NC 4.0) license, which permits others to distribute, remix, adapt, build upon this work noncommercially, and license their derivative works on different terms, provided the original work is properly cited and the use is non-commercial. See: http:// creativecommons.org/licenses/by-nc/4.0/

\section{REFERENCES}

1. Coste J, Job-Spira N, Aublet-Cuvelier B, et al. Incidence of ectopic pregnancy. First results of a population-based register in France. Hum Reprod 1994;9:742-5.

2. Walker JJ. Ectopic pregnancy. Clin Obstet Gynecol 2007;50:89-99.

3. Juneau C, Bates GW. Reproductive outcomes after medical and surgical management of ectopic pregnancy. Clin Obstet Gynecol 2012;55:455-60.

4. Bruhat MA, Manhes H, Mage G, et al. Treatment of ectopic pregnancy by means of laparoscopy. Fertil Steril 1980;33:411-14.

5. Tanaka T, Hayashi $\mathrm{H}$, Kutsuzawa T, et al. Treatment of interstitial ectopic pregnancy with methotrexate: report of a successful case. Fertil Steril 1982;37:851-2.

6. Desroque D, Capmas P, Legendre G, et al. Fertility after ectopic pregnancy. J Gynecol Obstet Biol Reprod (Paris) 2010;39:395-400.
7. Hajenius PJ, Mol F, Mol BW, et al. Interventions for tubal ectopic pregnancy. Cochrane Database Syst Rev 2007;(1):CD000324.

8. de Bennetot M, Rabischong B, Aublet-Cuvelier B, et al. Fertility after tubal ectopic pregnancy: results of a population-based study. Fertil Steril 2012;98:1271-6.

9. Yao M, Tulandi T. Current status of surgical and nonsurgical management of ectopic pregnancy. Fertil Steril 1997:67:421-33.

10. Bangsgaard N, Lund CO, Ottesen B, et al. Improved fertility following conservative surgical treatment of ectopic pregnancy. BJOG 2003;110:765-70.

11. Turan V. Fertility outcomes subsequent to treatment of tubal ectopic pregnancy in younger Turkish women. J Pediatr Adolesc Gynecol 2011;24:251-5

12. Deffieux X, Morin Surroca M, Faivre E, et al. Tubal anastomosis after tubal sterilization: a review. Arch Gynecol Obstet 2011;283:1149-58

13. Pouly JL, Chapron $\mathrm{C}$, Manhes $\mathrm{H}$, et al. Multifactorial analysis of fertility after conservative laparoscopic treatment of ectopic pregnancy in a series of 223 patients. Fertil Steril 1991;56:453-60.

14. Hajenius PJ, Mol BWJ, Ankum WM, et al. Clearance curves of serum human chorionic gonadotrophin for the diagnosis of persistent trophoblast. Hum Reprod 1995;10:683-7.

15. Nama V, Manyonda I. Tubal ectopic pregnancy: diagnosis and management. Arch Gynecol Obstet 2009;279:443.

16. Fernandez $\mathrm{H}$, Yves Vincent SC, Pauthier S, et al. Randomized trial of conservative laparoscopic treatment and methotrexate administration in ectopic pregnancy and subsequent fertility. Hum Reprod 1998;13:3239-43.

17. KragMoeller LB, Moeller C, Thomsen SG, et al. Success and spontaneous pregnancy rates following systemic methotrexate versus laparoscopic surgery for tubal pregnancies: a randomized trial. Acta Obstet Gynecol Scand 2009;88:1331-7.

18. Clausen I. Conservative versus radical surgery for tubal pregnancy A review. Acta Obstet Gynecol Scand 1996:75:8-12.

19. Silva PD, Schaper AM, Rooney B. Reproductive outcome after 143 laparoscopic procedures for ectopic pregnancy. Obstet Gynecol 1993;81:710-15.

20. Mol F, Mol BW, Ankum WM, et al. Current evidence on surgery, systemic methotrexate and expectant management in the treatment of tubal ectopic pregnancy: a systematic review and meta-analysis. Hum Reprod Update 2008;14:309.

21. Becker S, Solomayer E, Hornung R, et al. Optimal treatment for patients with ectopic pregnancies and a history of fertility-reducing factors. Arch Gynecol Obstet 2011;283:41.

22. Kim SH, Shin CJ, Kim JG, et al. Microsurgical reversal of tubal sterilization: a report on 1118 cases. Fertil Steril 1997;68:865-70.

23. Koh $\mathrm{CH}$, Janik GM. Laparoscopic microsurgical tubal anastomosis: results of 40 consecutive cases. In: Program and Abstracts of the 52nd Annual Meeting of the American Society for Reproductive Medicine. Boston, MA. 1996

24. Tan HH, Loh SF. Microsurgical reversal of sterilisation-is this still clinically relevant today? Ann Acad Med Singapore 2010;39: 22-6.

25. Kuroda K, Takeuchi H, Kitade M, et al. Assessment of tubal disorder as a risk factor for repeated ectopic pregnancy. $J$ Obstet Gynaecol Res 2009;35:520-4. 